Check for updates

Cite this: Chem. Commun., 2019, 55, 14906

Received 28th October 2019 Accepted 15th November 2019

DOI: $10.1039 /$ c9cc08423a

rsc.li/chemcomm

\section{Selenoamides modulate dipole-dipole interactions in hydrogen bonded supramolecular polymers of 1,3,5-substituted benzenes $\dagger$}

\author{
José Augusto Berrocal, (D) $\ddagger^{a}$ Mathijs F. J. Mabesoone, (D) $\ddagger^{a}$ \\ Miguel García Iglesias, (DD ${ }^{a b c}$ Alex Huizinga, ${ }^{a}$ E. W. Meijer (D) ${ }^{* a}$ and \\ Anja R. A. Palmans (D) *a
}

We report the synthesis and self-assembly behavior of a chiral $C_{3}$-symmetrical benzene-tricarboselenoamide. The introduction of the selenoamide moiety enhances the dipolar character of the supramolecular interaction and confers a remarkable thermal stability to the supramolecular polymers obtained.

Supramolecular chemistry has maturated up to a point where parallelisms between sophisticated multi-step covalent syntheses and the construction of increasingly complex, artificial supramolecular systems become more and more apparent. ${ }^{1-4}$ Recent developments in living supramolecular polymerizations, ${ }^{5-8}$ controlled supramolecular copolymerizations ${ }^{9-12}$ and the design of unconventional thermal behaviour in supramolecular polymers ${ }^{13-15}$ resulted in unprecedented control in length and microstructure of the formed polymers. Like in proteins, one of the central interactions underlying the stability and structure of many supramolecular systems is the formation of hydrogen bonds between amides. This ubiquitous interaction comprises several contributions such as dispersion interactions, electrostatics, polarization and charge transfer. ${ }^{16}$ Recently, it has become evident that the replacement of oxygen in amide bonds by other chalcogens such as sulphur and selenium retains the ability of the resulting thio- and selenoamides to engage in hydrogen bonding interactions. ${ }^{17-20}$ In addition, it has been observed both theoretically and experimentally that $\mathrm{NH} \cdots \mathrm{S}$ or $\mathrm{NH} \cdots$ Se hydrogen bonds can be as strong as those of $\mathrm{NH} \cdots \mathrm{O}$, despite the lower electronegativity of $S$ and $S e .^{21,22}$ In proteins

\footnotetext{
${ }^{a}$ Institute for Complex Molecular Systems, Eindhoven University of Technology, P. O Box 513, 5600 MB Eindhoven, The Netherlands. E-mail: a.palmans@tue.nl, e.w.meijer@tue.nl

${ }^{b}$ Department of Organic Chemistry, Universidad Autónoma de Madrid (UAM), Calle Francisco Tomás y Valiente, 7, 28049 Madrid, Spain ${ }^{c}$ IMDEA Nanociencia, c/Faraday 9, Cantoblanco, Spain $\dagger$ Electronic supplementary information (ESI) available: Experimental details, synthesis and characterization of all new compounds, spectroscopic details, modeling details, temperature- and solvent-dependent supramolecular polymerization studies and fits. See DOI: 10.1039/c9cc08423a

\$ These authors have contributed equally.
}

and peptides, the introduction of thioamide or selenoamide bonds has allowed to tune their structure, stability, and - in case of enzymes - activity. ${ }^{23-26}$ Interestingly, as the chalcogen atom in the amide in peptides changes from $\mathrm{O}$ to $\mathrm{S}$ to $\mathrm{Se}$, the increasing polarizability was found to increasingly dominate the electronic properties of the amides. As a result, the amide structure becomes increasingly characterized by the resonance form with negative charge on $\mathrm{S}$ or Se upon going down the periodic table. ${ }^{27}$ While the replacement of oxygen with sulphur typically results in a larger effect, the consequence of the further S to Se substitution is more modest, yet still detectable. As charge separation and dipolar interactions have been linked to cooperative behaviour in supramolecular polymers, ${ }^{28}$ selenoamides may provide interesting functionality in these noncovalent structures.

In the past we have extensively investigated the self-assembly of $C_{3}$-symmetrical $N, N^{\prime}, N^{\prime \prime \prime}$-trialkylbenzene-1,3,5-tricarboxamides (OBTAs) in apolar alkane solvents (achiral OBTA and chiral c-OBTA, Scheme 1) and more recently also of $N, N^{\prime}, N^{\prime \prime \prime}$ trialkylbenzene-1,3,5-trithioamides (S-BTAs) (achiral SBTA and c-SBTA, Scheme 1). ${ }^{29,30}$

Stimulated by the increased dipolar character of the interactions between thioamides as compared to normal amides, ${ }^{31-33}$ we hypothesized that moving one row further down the periodic

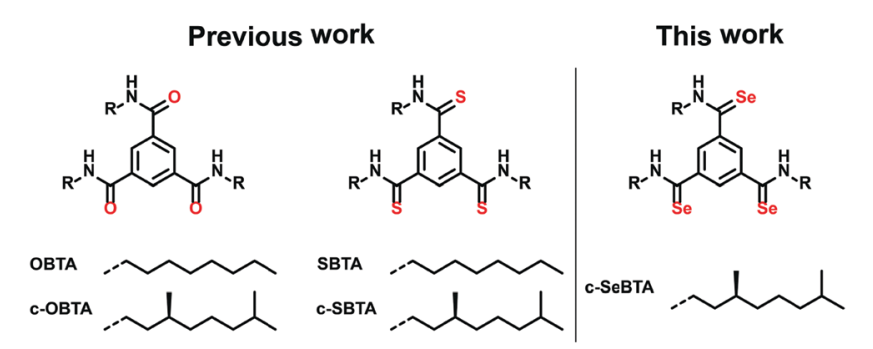

Scheme 1 Chemical structures of previously reported OBTA, c-OBTA, SBTA and C-SBTA; chemical structure of the chiral selenoamide derivative C-SeBTA introduced in this work. Enantiopure compounds are indicated with the prefix $\mathrm{C}^{-}$. 
table to selenoamides would affect the stability of the formed cooperative supramolecular polymers. In addition, we anticipated that in copolymerizations the microstructure of the copolymers could be modulated.

Here, we disclose the synthesis (reported in the ESI $\dagger$ ) and selfassembly of the chiral, $C_{3}$-symmetrical benzene-tricarboselenoamide c-SeBTA (Scheme 1). We compare its self-assembly behavior to that of OBTA/C-OBTA ${ }^{20}$ and SBTA/c-SBTA. ${ }^{21}$ The different nature of the selenoamide hydrogen bond is illustrated by our finding that c-SeBTA polymers have an increased stability towards thermal denaturation yet enhanced lability towards polar solventsinduced denaturation.

The chiral tricarboselenoamide c-SeBTA was synthesized in $82 \%$ yield by refluxing c-OBTA with Woollins reagent $\left(\mathrm{Ph}_{2} \mathrm{P}_{2} \mathrm{Se}_{4}\right)$ in toluene (Scheme S1, ESI $\dagger$ ). ${ }^{34}$ We monitored the successful conversion to selenoamides by Fourier-transform infrared (FT-IR) spectroscopy. The disappearance of the parent carboxamide $\mathrm{C}=\mathrm{O}$ vibration at $1634 \mathrm{~cm}^{-1}$ was coupled to the appearance of a new vibration centered at $1538 \mathrm{~cm}^{-1}$, indicative for the formation of the desired product (Fig. S3-S5, ESI $\dagger$ ). The N-H stretching vibration also shifted from $3220 \mathrm{~cm}^{-1}$ to $3151 \mathrm{~cm}^{-1}$ in the c-OBTA to c-SeBTA conversion. Further details on the molecular characterization of c-SeBTA can be found in the ESI. $\dagger$

Chiral SeBTA was obtained as a yellow solid. The yellow color remained upon storing the compound under argon. However, exposing the samples to air for several hours resulted in an irreversible color change (Fig. S6, ESI $\dagger$ ), suggesting decomposition. The low air stability of c-SeBTA was also coupled to poor thermal stability in ambient atmospheres, as we could not detect any transition upon heating or cooling due to decomposition of the samples by differential scanning calorimetry (DSC). Traces of c-OBTA detected in mass spectrometry suggest that c-SeBTA can react with moisture to form c-OBTA and other degradation products. Hence, the conversion of the tricarboxamides into triselenoamides implies particular attention concerning the compound's storage and preservation (under argon and preferably refrigerated).

We first studied the self-assembly behavior of c-SeBTA in apolar alkane solvents with ultraviolet-visible (UV-Vis) and circular dichroism (CD) spectroscopy. Upon dissolution of c-SeBTA in methylcyclohexane (MCH) at $50 \mu \mathrm{M}$ and thorough degassing, an absorption maximum at $342 \mathrm{~nm}$ was observed in the UV-vis spectrum (Fig. 1a, bottom part). This maximum was red-shifted with respect to the spectrum of c-SeBTA in methanol (Fig. 1a, bottom part), suggesting self-assembly in the alkane solvent. In addition, a bisignate CD spectrum was obtained in $\mathrm{MCH}$ (maxima/minima at $357 \mathrm{~nm}, 297 \mathrm{~nm}$ and $252 \mathrm{~nm}$ ), which corroborated the presence of aggregates characterized by a preferred helical sense (Fig. 1a, top part). AFM measurements confirmed that aggregates of c-SeBTA are one-dimensional supramolecular polymers (Fig. S7, ESI $\dagger$ ).

We employed variable temperature CD (VT-CD) spectroscopy to investigate the stability of the supramolecular polymers of c-SeBTA. We followed the intensity of the CD band centered at $357 \mathrm{~nm}$ (Fig. 1b). Careful degassing of the prepared solutions was pivotal to ensure the reproducibility of the experiments
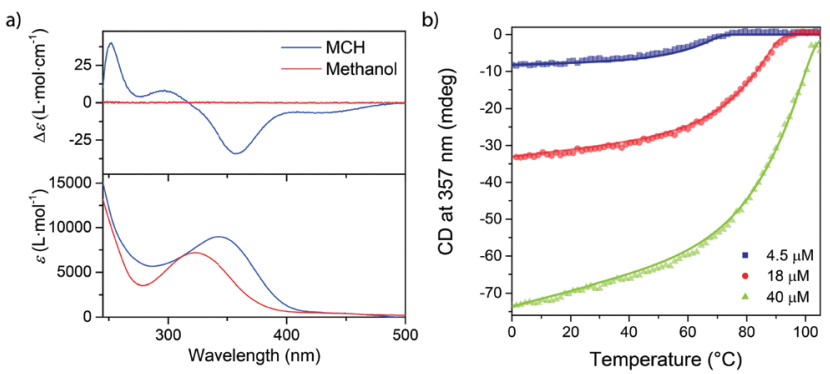

Fig. 1 (a) CD (top) and UV-vis (bottom) spectra of $50 \mu \mathrm{M}$ solutions of c-SeBTA in MCH and methanol (blue and red trace, respectively). (b) Variable temperature CD spectroscopy of solutions of c-SeBTA in MCH. Symbols indicate data and the solid lines indicate the global fit.

(for the degassing procedure see $\mathrm{ESI} \dagger$ ). The $40 \mu \mathrm{M}$ solutions of c-SeBTA showed CD activity up to temperatures as high as $100{ }^{\circ} \mathrm{C}$, indicating the high thermal stability of the generated supramolecular polymers (Fig. 1b, green trace). Upon decreasing the concentration to $18 \mu \mathrm{M}$ (Fig. $1 \mathrm{~b}$, red trace) and $4.5 \mu \mathrm{M}$ (Fig. 1b, blue trace), a sharp transition between the CD-silent monomerically dissolved c-SeBTA to the supramolecular polymers of c-SeBTA was observed at $93{ }^{\circ} \mathrm{C}$ and $75{ }^{\circ} \mathrm{C}$, respectively. This indicated a polymerization process characterized by a cooperative mechanism. By fitting the experimental data to a nucleationelongation model, ${ }^{35}$ we determined enthalpy $\left(\Delta H_{\mathrm{e}}\right)$ and entropy $\left(\Delta S_{\mathrm{e}}\right)$ of elongation equal to $-75 \mathrm{~kJ} \mathrm{~mole}^{-1}$ and $-97 \mathrm{~J} \mathrm{~mole}^{-1} \mathrm{~K}^{-1}$, respectively, and a nucleation penalty of $21 \mathrm{~kJ} \mathrm{~mole}^{-1}$. The latter corresponds to a cooperativity factor, $\sigma$, of $2 \times 10^{-4}$ at $293 \mathrm{~K}$. Compared to the supramolecular polymers of c-SBTA $\left(\Delta H_{\mathrm{e}}=\right.$ $-65.7 \mathrm{~kJ}$ mole $\left.^{-1}\right),{ }^{30,36}$ the enthalpic contribution in the supramolecular polymerization of c-SeBTA was slightly more favorable, suggesting stronger intermolecular interactions in the case of the selenoamides. The entropic penalty of the supramolecular polymerization of c-SeBTA $\left(\Delta S_{\mathrm{e}}=-97 \mathrm{~J} \mathrm{~mole}^{-1} \mathrm{~K}^{-1}\right)$ was comparable to that of c-SBTA ${ }^{30,36}\left(\Delta S_{\mathrm{e}}=-102.6 \mathrm{~J} \mathrm{~mole}^{-1} \mathrm{~K}^{-1}\right)$, instead, and we speculate similar ordering between the homopolymers of c-SeBTA and c-SBTA. We rationalize these thermodynamic parameters with an increased dipole-dipole and charge transfer character of the diffuse $\mathrm{Se}-\mathrm{NH}$ hydrogen bond, ${ }^{21,37}$ which ultimately affords the increased thermal stability of the supramolecular polymers of c-SeBTA (more favorable $\Delta H_{\mathrm{e}}$ and very similar $\Delta S_{\mathrm{e}}$ compared to c-SBTA). Interestingly, the nucleation penalty, and hence cooperativity, of the supramolecular polymers of c-SBTA and c-SeBTA were comparable $\left(\sigma=1.9 \times 10^{-3} \text { for c-SBTA }\right)^{30,36}$ and $3 \times 10^{-4}$ for c-SeBTA). This is in strong contrast to the lower cooperativity of c-SBTA compared to c-OBTA $\left(\sigma<10^{-6}\right){ }^{30,36}$

Next to VT-CD spectroscopy, the supramolecular polymerization of c-SeBTA was also investigated by changing the solvent quality in a denaturation experiment. Analogously to a previous study on $\mathbf{c - S B T A}{ }^{38}$ discrete aliquots of $\mathrm{CHCl}_{3}$ (good solvent) were added to $20 \mu \mathrm{M}$ solutions of c-SeBTA in $\mathrm{MCH}$ and the intensity of the CD signal was monitored (Fig. 2a and b). Upon increasing the solvent quality by adding $\mathrm{CHCl}_{3}$, the $\mathrm{CD}$ effect decreased in intensity (Fig. 2a). Above 4 vol\%, no CD intensity could be observed anymore, indicating the complete destabilization of the polymers in 96:4 $\mathrm{MCH}: \mathrm{CHCl}_{3}$ (Fig. 2a). When monitoring the 

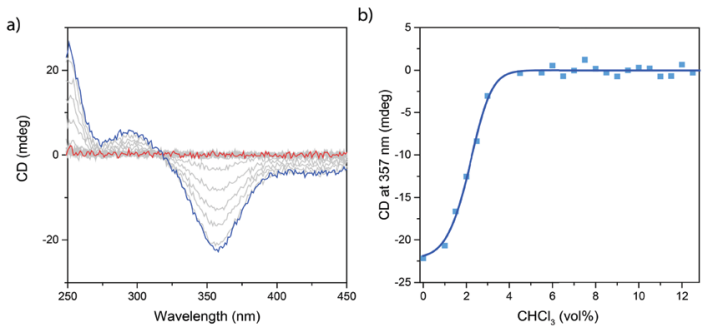

Fig. 2 Denaturation experiment of c-SeBTA. (a) Change of the CD spectra of c-SeBTA (20 $\mu \mathrm{M}$ in $\mathrm{MCH}$, blue line) upon adding $\mathrm{CHCl}_{3}(0-12$ vol\%, red line) and (b) $\mathrm{CD}$ effect at $357 \mathrm{~nm}$ as a function of the amount of $\mathrm{CHCl}_{3}$. The squares indicate experimental data and the solid lines represent the simulation.

maximum CD intensity at $357 \mathrm{~nm}$, a clear non-sigmoidal dependency on the solvent quality is obtained (Fig. 2b), confirming the cooperative polymerization mechanism observed in the VT-CD experiments. The critical solvent fraction of $4 \mathrm{vol} \% \mathrm{CHCl}_{3}$ in $\mathrm{MCH}$ at $20 \mu \mathrm{M}$ for c-SeBTA is in remarkable contrast with a critical $\mathrm{CHCl}_{3}$ fraction of 20 vol\% obtained for c-SBTA. ${ }^{38}$ This was unexpected, given the similar thermal behavior of the supramolecular polymers of c-SeBTA and c-SBTA discussed above. To obtain more insights into the thermodynamic properties of the denaturation process, the spectroscopic data were fitted to a mass-balance model that describes the destabilization of supramolecular polymers. ${ }^{38}$ For c-SeBTA, we obtain a Gibbs free energy of elongation $\left(\Delta G_{\mathrm{e}}^{\mathrm{den}}\right)$ of $-36 \mathrm{~kJ} \mathrm{~mole}^{-1}$ in $\mathrm{MCH}$, a solvent dependency parameter $(m)$ of $602 \mathrm{~kJ} \mathrm{~mole}^{-1}$, as well as a nucleation penalty of $2.6 \mathrm{~kJ} \mathrm{~mole}^{-1}$. This Gibbs free energy of elongation is comparable to the Gibbs free energy of $-40 \mathrm{~kJ}$ mole ${ }^{-1}$ reported for c-SBTA, but the solvent dependency parameter, $m$, obtained for c-SeBTA is six fold higher than the $103 \mathrm{~kJ} \mathrm{~mole}^{-1}$ reported for c-SBTA. ${ }^{38} \mathrm{We}$ conclude that the enhanced charge separation in selenoamides ${ }^{27}$ increases the solubility in the relatively polar $\mathrm{CHCl}_{3}$ solvent. As a result, the interaction of the monomer with the polar co-solvent is enhanced and smaller amounts of co-solvent are needed to completely denature the polymer. Thus, on one side installing the selenoamide motif increases the thermal stability of the resulting supramolecular polymers in pure alkane solvents, while it simultaneously decreases their stability in more polar environments.

Besides influencing the stabilities of the supramolecular homopolymers, the different polarizability of oxygen, sulfur and selenium offers attractive possibilities to tune the interactions between monomers in supramolecular copolymers. We carried out mixed sergeants and soldiers experiment using c-SeBTA as sergeant for OBTA and SBTA to investigate the interaction between these supramolecular monomers (Fig. 3). The gradual titration of chiral c-SeBTA to a $30 \mu \mathrm{M}$ solution of achiral OBTA in $\mathrm{MCH}$ resulted in the appearance of a $\mathrm{CD}$ spectrum typically observed for c-OBTA (Fig. S9a, ESI $\dagger$ ), indicating that OBTA and c-SeBTA form copolymers. Complete chiral amplification was obtained by adding more than $30 \mathrm{~mol} \%$ of c-SeBTA, as suggested by the saturation of the CD signal with respect to the intensity expected for a $30 \mu \mathrm{M}$ solution of c-OBTA (Fig. 3 top panel). Analogously, addition of c-SeBTA to a $30 \mu \mathrm{M}$ solution of SBTA resulted in the appearance of a CD spectrum

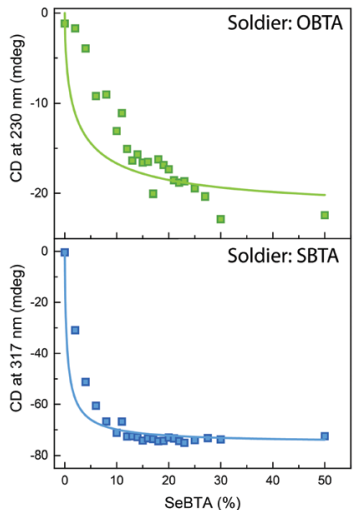

Fig. 3 Sergeants and soldiers experiment of c-SeBTA sergeants with 30 mol\% OBTA soldiers (top panel) and SBTA soldiers (bottom panel) in $\mathrm{MCH}$. The squares indicate experimental data and the solid lines represent the simulation.

typically observed for c-SBTA, ${ }^{36}$ which indicated successful copolymerization (Fig. S9b, ESI $\dagger$ ). c-SeBTA was more effective in biasing the helical sense in the case of SBTA: only $12 \mathrm{~mol} \%$ c-SeBTA was required to achieve full chiral amplification (Fig. 3 bottom panel). We applied the model developed by Markvoort and ten Eikelder ${ }^{10,39}$ to quantify the heterointeraction of c-SeBTA with OBTA and SBTA in the sergeants and soldiers experiment. These heterointeractions are dependent on the two homopolymerizations through an $\alpha$-parameter (eqn (1)), ${ }^{30}$

$$
\Delta G_{\text {hetero }}=\frac{1}{2} \cdot \alpha \cdot\left(\Delta G_{\mathrm{e}, \mathrm{SeBTA}}+\Delta G_{\mathrm{e}, \mathrm{XBTA}}\right)
$$

with $\Delta G_{\text {hetero }}$ the Gibbs free energy of the interaction between c-SeBTA and OBTA or SBTA, $\Delta G_{\mathrm{e}, \mathrm{SeBTA}}$ the Gibbs free energy of the elongation of the c-SeBTA homopolymer, and $\Delta G_{\mathrm{e}, \mathrm{XBTA}}$ the Gibbs free energy of the elongation of the OBTA or SBTA homopolymer.

The thermodynamic values for OBTA and SBTA have been described elsewhere. ${ }^{30}$ We obtained a good agreement between our experimental results and the model prediction for SBTA/ c-SeBTA when using $\alpha=0.815$ (Fig. 3 bottom panel and Table 1). This value is lower than that previously reported for the nearly random copolymers of SBTA/OBTA $(\alpha=0.92)^{29}$ but higher than those of the blocky copolymers formed by triarylamine-based systems $(\alpha=0.57) .{ }^{10}$ This indicates that the copolymers of c-SeBTA/SBTA have a slightly blocky microstructure. In contrast, we could not find a good agreement between the model and the experimental data of OBTA/c-SeBTA (best fit for $\alpha=0.72$, Fig. 3 top panel). Most likely, the assumption made in the model that $\Delta G_{\text {hetero }}$ is identical for $\mathrm{O}=\mathrm{CNH} \cdots \mathrm{Se}=\mathrm{C}$ and $\mathrm{Se}=\mathrm{CNH} \cdots \mathrm{O}=\mathrm{C}$

Table 1 Thermodynamic values $\left(20{ }^{\circ} \mathrm{C}\right)$ showing good agreement between model predictions and experimental results with c-SeBTA sergeants and OBTA/SBTA soldiers

\begin{tabular}{lcc}
\hline & OBTA & SBTA \\
\hline$\Delta G_{\mathrm{e}, \text { XBTA }}\left(\mathrm{kJ} \mathrm{mole}^{-1}\right)$ & -33.6 & -36.4 \\
$\alpha$ & 0.72 & 0.815 \\
$\Delta G_{\text {hetero }}\left(\mathrm{kJ} \mathrm{mole}^{-1}\right)$ & -28.9 & -33.8
\end{tabular}


does not hold. The energetic difference between the XBTA homopolymerization ( $\mathrm{X}=\mathrm{O}$ or $\mathrm{S}$ ) and the heterointeraction was smaller for the c-SeBTA/SBTA couple compared to the c-SeBTA/OBTA one (Table 1). The smaller difference in interaction strength for the neighboring chalcogens suggests that it is the polarizability of the groups that governs the interaction strength between the different monomers in the copolymerization. Hence, tuning heterointeractions by means of polarizability of the interacting co-monomers may prove to be an attractive way to tune supramolecular copolymerizations and control the microstructure of the resulting copolymers.

In conclusion, similarly to their oxygen and sulfur analogues, selenoamide-based discotic molecules self-assemble through hydrogen bond formation in apolar alkane solvents. The introduction of the selenoamides further increases the dipole-dipole and charge transfer interactions in the hydrogen bonding and continues a trend which was already observed upon changing carboxamides to thioamides. ${ }^{36}$ As a result, c-SeBTA shows an enhanced stability towards thermal denaturation in apolar alkane solvents while also showing an increased sensitivity towards polar co-solvents. Additionally, the subtle role played by polarizability and dipole-dipole interactions in hydrogen bonds offers an attractive way to design and control supramolecular copolymers. We showed successful copolymerization of c-SeBTA with OBTA and SBTA through sergeants and soldiers experiments. The interaction strength is highest for neighboring chalcogens, leading to the possibility to tune the supramolecular copolymerization through modulation of the dipole-dipole and charge transfer interactions in hydrogen bonds. We propose that our results will allow further control in the design of novel supramolecular polymers and serve as inspiration for further theoretical and experimental investigations into selenoamide hydrogen bonds.

Lafayette de Windt is gratefully acknowledged for providing the achiral OBTA and SBTA and for fruitful discussions. Tristan Mes and Elisa Huerta are acknowledged for the initial experiments. The authors thank NWO for financial support.

\section{Conflicts of interest}

There are no conflicts to declare.

\section{Notes and references}

1 N. Giuseppone, Acc. Chem. Res., 2012, 45, 2178-2188.

2 J. Lehn, Angew. Chem., Int. Ed., 2013, 52, 2836-2850.

3 P. Besenius, J. Polym. Sci., Part A: Polym. Chem., 2017, 55, 34-78.

4 G. Vantomme and E. W. Meijer, Science, 2019, 363, 1396-1397.

5 S. Ogi, K. Sugiyasu, S. Manna, S. Samitsu and M. Takeuchi, Nat. Chem., 2014, 6, 188-195.

6 T. Fukui, S. Kawai, S. Fujinuma, Y. Matsushita, T. Yasuda, T. Sakurai, S. Seki, M. Takeuchi and K. Sugiyasu, Nat. Chem., 2017, 9, 493-499.

7 W. Wagner, M. Wehner, V. Stepanenko, S. Ogi and F. Würthner, Angew. Chem., Int. Ed., 2017, 56, 16008-16012.
8 J. S. Valera, R. Gómez and L. Sánchez, Small, 2018, 14, 1702437.

9 A. Pal, M. Malakoutikhah, G. Leonetti, M. Tezcan, M. ColombDelsuc, V. D. Nguyen, J. Van Der Gucht and S. Otto, Angew. Chem., Int. Ed., 2015, 54, 7852-7856.

10 B. Adelizzi, A. Aloi, A. J. Markvoort, H. M. M. Ten Eikelder, I. K. Voets, A. R. A. Palmans and E. W. Meijer, J. Am. Chem. Soc., 2018, 140, 7168-7175.

11 S. H. Jung, D. Bochicchio, G. M. Pavan, M. Takeuchi and K. Sugiyasu, J. Am. Chem. Soc., 2018, 140, 10570-10577.

12 S. Chakraborty, D. Ray, V. K. Aswal and S. Ghosh, Chem. - Eur. J., 2018, 24, 16379-16387.

13 T. Vermonden, J. Van Der Gucht, P. De Waard, A. T. M. Marcelis, N. A. M. Besseling, E. J. R. Sudhölter, G. J. Fleer and M. A. Cohen Stuart, Macromolecules, 2003, 36, 7035-7044.

14 K. Venkata Rao, D. Miyajima, A. Nihonyanagi and T. Aida, Nat. Chem., 2017, 9, 1133-1139.

15 V. Grande, B. Soberats, S. Herbst, V. Stepanenko and F. Würthner, Chem. Sci., 2018, 9, 6904-6911.

16 T. Steiner, Angew. Chem., Int. Ed., 2002, 41, 48-76.

17 C. Alemán, J. Phys. Chem. A, 2001, 105, 6717-6723.

18 H.-J. Lee, Y.-S. Choi, K.-B. Lee, J. Park and C.-J. Yoon, J. Phys. Chem. A, 2002, 106, 7010-7017.

19 D. Bibelayi, A. S. Lundemba, F. H. Allen, P. T. A. Galek, J. Pradon, A. M. Reilly, C. R. Groom and Z. G. Yav, Acta Crystallogr., Sect. B: Struct. Sci., Cryst. Eng. Mater., 2016, 72, 317-325.

20 V. R. Mundlapati, S. Gautam, D. K. Sahoo, A. Ghosh and H. S. Biswal, J. Phys. Chem. Lett., 2017, 8, 4573-4579.

21 K. K. Mishra, S. K. Singh, P. Ghosh, D. Ghosh and A. Das, Phys. Chem. Chem. Phys., 2017, 19, 24179-24187.

22 V. R. Mundlapati, D. K. Sahoo, S. Ghosh, U. K. Purame, S. Pandey, R. Acharya, N. Pal, P. Tiwari and H. S. Biswal, J. Phys. Chem. Lett., 2017, 8, 794-800.

23 T. T. Tran, J. Zeng, H. Treutlein and A. W. Burgess, J. Am. Chem. Soc., 2002, 124, 5222-5230.

24 D. Wildemann, C. Schiene-Fischer, T. Aumüller, A. Bachmann, T. Kiefhaber, C. Lücke and G. Fischer, J. Am. Chem. Soc., 2007, 129, 4910-4918.

25 Y. Huang, G. Jahreis, C. Lücke, D. Wildemann and G. Fischer, J. Am. Chem. Soc., 2010, 132, 7578-7579.

26 C. R. Walters, D. M. Szantai-Kis, Y. Zhang, Z. E. Reinert, W. S. Horne, D. M. Chenoweth and E. J. Petersson, Chem. Sci., 2017, 8, 2868-2877.

27 Y. Huang, G. Jahreis, G. Fischer and C. Lücke, Chem. - Eur. J., 2012, 18, 9841-9848.

28 C. Kulkarni, S. Balasubramanian and S. J. George, ChemPhysChem, 2013, 14, 661-673.

29 M. M. J. Smulders, A. P. H. J. Schenning and E. W. Meijer, J. Am. Chem. Soc., 2008, 130, 606-611.

30 L. N. J. de Windt, C. Kulkarni, H. M. M. ten Eikelder, A. J. Markvoort, E. W. Meijer and A. R. A. Palmans, Macromolecules, 2019, 52, 7430-7438.

31 O. Exner and K. Waisser, Collect. Czech. Chem. Commun., 1982, 47, 828-837.

32 K. B. Wiberg and P. R. Rablen, J. Am. Chem. Soc., 1995, 117, 2201-2209.

33 K. B. Wiberg and D. J. Rush, J. Am. Chem. Soc., 2001, 123, 2038-2046.

34 P. Bhattacharyya and J. D. Woollins, Tetrahedron Lett., 2001, 42, 5949-5951.

35 D. Zhao and J. S. Moore, Org. Biomol. Chem., 2003, 1, 3471-3491.

36 T. Mes, S. Cantekin, D. W. R. Balkenende, M. M. M. Frissen, M. A. J. Gillissen, B. F. M. De Waal, I. K. Voets, E. W. Meijer and A. R. A. Palmans, Chem. - Eur. J., 2013, 19, 8642-8649.

37 A. Kjaersgaard, J. R. Lane and H. G. Kjaergaard, J. Phys. Chem. A, 2019, 123, 8427-8434.

38 P. A. Korevaar, C. Schaefer, T. F. A. De Greef and E. W. Meijer, J. Am. Chem. Soc., 2012, 134, 13482-13491.

39 H. M. M. ten Eikelder, B. Adelizzi, A. R. A. Palmans and A. J. Markvoort, J. Phys. Chem. B, 2019, 123, 6627-6642. 